\title{
Investigations on mineral content in warty crab Eriphia verrucosa (Forskal, 1775) from the Rize Coast of Black Sea, Turkey
}

\author{
EMRE CAGLAK AND BARIS KARSLI \\ Recep Tayyip Erdogan University, Faculty of Fisheries, Department of Processing Technology, 53100, Rize, Turkey \\ e-mail: emre.caglak@erdogan.edu.tr
}

\begin{abstract}
This study investigated the seasonal variations in mineral content of edible parts of male and female warty crab Eriphia verrucosa (Forskal, 1775) distributed along Rize Coast of Black Sea and compared the same with the limit values of mineral content for human consumption set by various national and international standards. The contents of $\mathrm{Al}, \mathrm{B}, \mathrm{Ca}, \mathrm{Cd}, \mathrm{Cr}, \mathrm{Cu}$, $\mathrm{Fe}, \mathrm{K}, \mathrm{Mg}, \mathrm{Mn}, \mathrm{Mo}, \mathrm{Na}$, Ni and $\mathrm{P}$ estimated during the present study did not exceed the limit values as per the national and international standards. However, $\mathrm{Pb}\left(0.25-0.70 \mathrm{mg} \mathrm{kg}^{-1}\right)$, Se $\left(0.29-0.75 \mathrm{mg} \mathrm{kg}^{-1}\right)$ and $\mathrm{Zn}\left(43.86-72.59 \mathrm{mg} \mathrm{kg}^{-1}\right) \mathrm{contents}^{-}$ were found to be above the limit values. In terms of daily consumption values, it was found that, $\mathrm{Al}\left(1.86-3.35 \mathrm{mg} \mathrm{kg}^{-1}\right)$, $\mathrm{B}\left(2.17-3.65 \mathrm{mg} \mathrm{kg}^{-1}\right), \mathrm{Fe}\left(2.80-6.44 \mathrm{mg} \mathrm{kg}^{-1}\right)$ and $\mathrm{Mo}\left(0.01-0.02 \mathrm{mg} \mathrm{kg}^{-1}\right)$ contents were within the limits as per all standards; while $\mathrm{Ca}, \mathrm{Cd}, \mathrm{Cr}, \mathrm{K}, \mathrm{Mg}, \mathrm{Mn}, \mathrm{Na}$ and $\mathrm{P}$ contents were above the limits prescribed by certain standards and $\mathrm{Cu}, \mathrm{Pb}, \mathrm{Se}$ and $\mathrm{Zn}$ contents were above the limits as per all the standards Most mineral contents of female crabs were higher than those of the males. Additionally, mineral content of the crabs was observed to increase during summer compared to other seasons.
\end{abstract}

Keywords: Black Sea, Eriphia verrucosa, Gender, Mineral content, Season, Warty crab

\section{Introduction}

Seafoods are one of the most important sources of healthy and balanced diet of which crustaceans are of significant economic importance due to the quality of edible meat. Crab meat is said to be an excellent source in terms of macro and micro elements such as calcium, iron, zinc, potassium, phosphorus, copper, manganese, zinc and calcium, in particular (Kucukgelmez et al., 2006; Chen et al., 2007; Kayhan et al., 2010; Jimmy and Arazu 2012).

The warty crab Eriphia verrucosa (Forskal, 1775) is an important species of crab in the Black Sea which is mostly consumed in the Aegean and Mediterranean coasts in Turkey. Total production of warty crab in Turkey in 2016 was $6 \mathrm{t}$ (TUIK, 2016). The present study attempted to estimate the content of minerals. viz., aluminum (Al), boron $(\mathrm{B})$, calcium $(\mathrm{Ca})$, cadmium $(\mathrm{Cd})$, chromium $(\mathrm{Cr})$, copper $(\mathrm{Cu})$, iron $(\mathrm{Fe})$, potassium $(\mathrm{K})$, magnesium $(\mathrm{Mg})$, manganese $(\mathrm{Mn})$, molybdenum $(\mathrm{Mo})$, sodium $(\mathrm{Na})$, nickel $(\mathrm{Ni})$, phosphorus $(\mathrm{P})$, lead $(\mathrm{Pb})$, selenium $(\mathrm{Se})$ and zinc $(\mathrm{Zn})$ in the warty crab caught in the Rize coast of the Black Sea. Nutritional value of crab meat in terms of human health and the metal pollution of the region were also assessed during the study.

\section{Materials and methods}

Study area and sampling

The study was carried out druing the period May 2012 to March 2013 in coastal areas of Rize Province in the Eastern Black Sea coast (between $41.05 \mathrm{~N}-40.46 \mathrm{E}$, $41.03 \mathrm{~N}-40.45 \mathrm{E}, 41.05 \mathrm{~N}-40.62 \mathrm{E}, 41.06 \mathrm{~N}-40.60 \mathrm{E}$ coordinates). The crab samples for the study were collected from fishermen engaged in fishing, operating nets in the depth range of 10-20 m.

A total of 103 crab samples were used in the study. The samples taken seasonally were kept alive in styrofoam boxes with ice and brought to the Processing Technology Laboratory, Faculty of Fisheries, Recep Tayyip Erdogan University, Turkey. Crab samples were sorted into females and males, their length and weight measurements were taken using a digital caliper (Maher 16; with a precision of $0.1 \mathrm{~mm}$ ) and a digital scale (And G-200 with a precision of $0.01 \mathrm{~g}$ ) respectively. The carapace meat and claw meat of the crabs were picked by hand, pooled and the meat thus collected from 40 females (average weight $78.39 \pm 18.59 \mathrm{~g}$ ) and 63 males (average weight $156.98 \pm 29.84 \mathrm{~g}$ ) were labeled and preserved at $72^{\circ} \mathrm{C}$ until analysis.

\section{Mineral content analysis}

The crab meat samples were dried at $105^{\circ} \mathrm{C}$, crushed in a porcelain mortar and $0.5 \mathrm{~g}$ sample was treated with $5 \mathrm{ml}$ of nitric acid $(65 \%)$ at room temperature for $24 \mathrm{~h}$ followed by incubation at $100^{\circ} \mathrm{C} 4 \mathrm{~h}$. The acid treated samples were cooled to room temperature and filtered (Whatman no. 42). The filtered samples were made up to $25 \mathrm{ml}$ with deionised water (Caglak and Karsl1, 2014) and mineral contents were analysed in an inductively coupled 
plasma optical emission spectrometer (Optima ICP-OES 7000 DV, Perkin Elmer). The sample preparation for analysis was carried out as per FAO technical report 158 (Bernhard, 1976; Zhang et al., 2007; Sukender et al., 2012). The wavelength frequencies (absorbance values) used for different elements were: Al - 396,153 nm, B - 249,677 nm, $\mathrm{Ca}-317,933 \mathrm{~nm}, \mathrm{Cd}-228,802 \mathrm{~nm}, \mathrm{Cr}-267,716 \mathrm{~nm}$, $\mathrm{Cu}-327,393 \mathrm{~nm}, \mathrm{Fe}-238,204 \mathrm{~nm}, \mathrm{~K}-766,490 \mathrm{~nm}$, $\mathrm{Mg}-285,213 \mathrm{~nm}, \mathrm{Mn}-257,610 \mathrm{~nm}$, Mo $-202,031 \mathrm{~nm}$, $\mathrm{Na}-589,592 \mathrm{~nm}, \mathrm{Ni}-231,604 \mathrm{~nm}, \mathrm{P}-213,617 \mathrm{~nm}$, $\mathrm{Pb}-220,353 \mathrm{~nm}$, Se - 196,026 nm and Zn - 206,200 nm. The concentration of elements were calculated from dry weight and expressed as $\mathrm{mg} \mathrm{kg}^{-1}$ wet weight.

\section{Statistical analysis}

SPSS 11.0 (SPSS, Inc., Chicago, IL, USA) software package was used for statistical analysis of the data. In order to test the significance of difference in mineral contents between males and females as well as between seasons, one way ANOVA and least significant difference 'LSD' test were applied $(\mathrm{p}<0.05)$ (Sumbuloglu and Sumbuloglu, 2000).

\section{Results and discussion}

Mineral contents of male and female crabs in different seasons recorded during the present study are presented in Table 1. Table 2 summarises the mineral content recorded in selected marine mollusc/crustacean species from different locations. The limit values for these minerals for human consumption set by various national and international standards are given in Table 3 and 4 .

\section{Aluminum (Al)}

The lowest Al content observed in the present study was $1.86 \mathrm{mg} \mathrm{kg}^{-1}$ recorded in male specimens in spring season. The highest amount (3.35 $\left.\mathrm{mg} \mathrm{kg}^{-1}\right)$ was found in females in summer. Al levels recorded in the literature ranged between $1.2-58.7 \mathrm{mg} \mathrm{kg}^{-1}$ (Mutlu et al., 2011; Bordon et al., 2012). As per the Agency for Toxic Substances and Disease Registry, USA (ATSDR, 2013) and the Joint FAO/WHO Expert Committee on Food Additives (JECFA, 1989), the consumption limit of $\mathrm{Al}$ is $1 \mathrm{mg} \mathrm{kg}^{-1} \mathrm{day}^{-1}$. Considering the average human weight of about $60-70 \mathrm{~kg}$, Al content of warty crabs recorded during the present study has been found to pose no risk for human consumption.

\section{Boron (B)}

The highest value of boron (B) $\left(3.65 \mathrm{mg} \mathrm{kg}^{-1}\right)$ was found in female crabs in summer, while the lowest value of $2.17 \mathrm{mg} \mathrm{kg}^{-1}$ was observed in males in autumn. Our results were found to be lower than the boron content of $6.70-19.89 \mathrm{mg} \mathrm{kg}^{-1}$ reported by Demopoulos et al. (2008) in Scylla serrata. Limit value of boron for human consumption has been specified as $0.2 \mathrm{mg} \mathrm{kg}^{-1} \mathrm{day}^{-1}$ (ATSDR, 2013) and the content recorded in warty crabs during the present study was not found to be at risk for consumption by human adults.

Table 1. Seasonal mineral contents of warty crab ( $\mathrm{mg} \mathrm{kg}^{-1}$ wet weight) (black: maximum, grey: minimum)

\begin{tabular}{|c|c|c|c|c|c|c|c|c|}
\hline \multirow{3}{*}{ Minerals } & \multicolumn{8}{|c|}{ Seasons } \\
\hline & \multicolumn{2}{|c|}{ Spring } & \multicolumn{2}{|c|}{ Summer } & \multicolumn{2}{|c|}{ Autumm } & \multicolumn{2}{|l|}{ Winter } \\
\hline & Male & Female & Male & Female & Male & Female & Male & Female \\
\hline $\mathrm{Al}$ & $1.86 \pm 0.60_{\mathrm{A}}^{\mathrm{a}}$ & $3.16 \pm 0.92_{\mathrm{A}}{ }^{\mathrm{a}}$ & $2.09 \pm 0.04_{\mathrm{A}}^{\mathrm{a}}$ & $3.35 \pm 0.14_{A}^{\sigma}$ & $2.59 \pm 0.11_{\mathrm{A}}^{\mathrm{a}}$ & $3.24 \pm 0.35_{\mathrm{A}}^{\mathrm{a}}$ & $2.17 \pm 0.05_{\mathrm{A}}^{\mathrm{a}}$ & $2.83 \pm 0.15_{\mathrm{A}}^{\mathrm{b}}$ \\
\hline B & $2.28 \pm 0.06_{\mathrm{A}}^{\mathrm{b}}$ & $2.52 \pm 0.37_{\mathrm{A}}^{\mathrm{a}}$ & $2.50 \pm 0.09_{\mathrm{A}}^{\mathrm{a}}$ & $3.65 \pm 0.42_{\mathrm{A}}^{\mathrm{a}}$ & $2.17 \pm 0.57_{\mathrm{A}}^{\mathrm{a}}$ & $3.54 \pm 1.38_{\mathrm{A}}{ }^{\mathrm{a}}$ & $2.29 \pm 0.15_{\mathrm{A}}^{\mathrm{a}}$ & $2.69 \pm 0.12_{\mathrm{A}}^{\mathrm{a}}$ \\
\hline $\mathrm{Ca}$ & $773.73 \pm 296.67_{\mathrm{AB}}^{\mathrm{a}}$ & $1102.62 \pm 408.45_{\mathrm{A}}^{\mathrm{a}}$ & $1419.19 \pm 2.34_{A}^{a}$ & $1364.89 \pm 193.09_{A}^{a}$ & $411.15 \pm 174.09_{\mathrm{B}}^{\mathrm{a}}$ & $1185.98 \pm 349.11_{A}{ }^{a}$ & $201.85 \pm 9.88_{B}{ }^{a}$ & $432.88 \pm 275.54_{A}{ }^{a}$ \\
\hline $\mathrm{Cd}$ & $0.02 \pm 0.01_{\mathrm{A}}^{\mathrm{a}}$ & $0.04 \pm 0.01_{\mathrm{A}}^{\mathrm{a}}$ & $0.03 \pm 0.00_{\mathrm{A}}^{\mathrm{a}}$ & $0.05 \pm 0.00_{B}^{b}$ & $0.03 \pm 0.01_{\mathrm{A}}^{\mathrm{a}}$ & $0.04 \pm 0.00_{\mathrm{AB}}^{\mathrm{a}}$ & $0.03 \pm 0.00_{\mathrm{A}}^{\mathrm{a}}$ & $0.03 \pm 0.00_{\mathrm{A}}^{\mathrm{a}}$ \\
\hline $\mathrm{Cr}$ & $0.05 \pm 0.02_{\mathrm{A}}^{\mathrm{a}}$ & $0.05 \pm 0.01_{\mathrm{A}}^{\mathrm{a}}$ & $0.15 \pm 0.11_{\mathrm{A}}^{\mathrm{a}}$ & $0.10 \pm 0.01_{A}^{a}$ & $0.31 \pm 0.04_{\mathrm{A}}^{\mathrm{a}}$ & $0.13 \pm 0.01_{\mathrm{A}}^{\mathrm{b}}$ & $0.35 \pm 0.23_{\mathrm{A}}^{\mathrm{a}}$ & $0.11 \pm 0.01_{\mathrm{A}}^{\mathrm{a}}$ \\
\hline $\mathrm{Cu}$ & $7.29 \pm 1.31_{\mathrm{A}}^{\mathrm{a}}$ & $8.03 \pm 0.06_{\mathrm{A}}^{\mathrm{a}}$ & $7.30 \pm 0.88_{\mathrm{A}}^{\mathrm{a}}$ & $7.91 \pm 1.10_{\mathrm{A}}^{\mathrm{a}}$ & $5.29 \pm 0.32_{\mathrm{A}}{ }^{\mathrm{a}}$ & $8.03 \pm 0.20_{\mathrm{A}}^{\mathrm{b}}$ & $5.12 \pm 0.20_{\mathrm{A}}^{\mathrm{a}}$ & $4.75 \pm 0.56_{B}{ }^{a}$ \\
\hline $\mathrm{Fe}$ & $2.80 \pm 0.75_{\mathrm{A}}^{\mathrm{a}}$ & $6.44 \pm 4.95_{A}^{a}$ & $2.64 \pm 0.31_{\mathrm{A}}^{\mathrm{a}}$ & $4.10 \pm 0.09_{\mathrm{A}}^{\mathrm{b}}$ & $3.37 \pm 0.25_{\mathrm{A}}{ }^{\mathrm{a}}$ & $5.26 \pm 1.62_{\mathrm{A}}^{\mathrm{a}}$ & $3.26 \pm 0.45_{\mathrm{A}}^{\mathrm{a}}$ & $2.86 \pm 0.14_{\mathrm{A}}^{\mathrm{a}}$ \\
\hline K & $1297.45 \pm 117.89_{\mathrm{A}}^{\mathrm{a}}$ & $1180.1 \pm 65.47_{\mathrm{A}}^{\mathrm{a}}$ & $1060.67 \pm 505.63_{A}^{a}$ & $1961.83 \pm 30.15_{B}^{a}$ & $1443.18 \pm 5.21_{\mathrm{A}}{ }^{\mathrm{a}}$ & $1811.17 \pm 116.37_{\mathrm{B}}^{\mathrm{b}}$ & $1368.45 \pm 10.08_{\mathrm{A}}{ }^{\mathrm{a}}$ & $1479.99 \pm 23.62_{C}^{b}$ \\
\hline $\mathrm{Mg}$ & $225.64 \pm 17.92_{\mathrm{A}}{ }^{\mathrm{a}}$ & $241.15 \pm 13.52_{\mathrm{AB}}{ }^{a}$ & $215.38 \pm 32.62_{\mathrm{A}}{ }^{\mathrm{a}}$ & $291.94 \pm 19.76_{A}{ }^{a}$ & $168.44 \pm 16.51_{\mathrm{A}}{ }^{\mathrm{a}}$ & $290.5 \pm 17.46_{\mathrm{A}}^{\mathrm{b}}$ & $156.84 \pm 5.09{ }_{\mathrm{A}}^{\mathrm{a}}$ & $188.63 \pm 16.07_{\mathrm{B}}^{\mathrm{a}}$ \\
\hline $\mathrm{Mn}$ & $0.50 \pm 0.06_{\mathrm{AB}}^{\mathrm{a}}$ & $1.93 \pm 0.41_{\mathrm{A}}^{\mathrm{b}}$ & $0.86 \pm 0.20_{\mathrm{A}}^{\mathrm{a}}$ & $1.75 \pm 0.24_{\mathrm{A}}^{\mathrm{a}}$ & $0.62 \pm 0.06_{\mathrm{AB}}^{\mathrm{a}}$ & $1.69 \pm 0.26_{\mathrm{A}}^{\mathrm{b}}$ & $0.32 \pm 0.01_{\mathrm{B}}^{\mathrm{a}}$ & $0.42 \pm 0.03_{\mathrm{B}}^{\mathrm{b}}$ \\
\hline Mo & $0.01 \pm 0.00_{\mathrm{A}}^{\mathrm{a}}$ & $0.01 \pm 0.00_{\mathrm{A}}^{\mathrm{a}}$ & $0.01 \pm 0.00_{\mathrm{A}}^{\mathrm{a}}$ & $0.01 \pm 0.01_{\mathrm{A}}^{\mathrm{a}}$ & $0.01 \pm 0.00_{\mathrm{A}}{ }^{\mathrm{a}}$ & $0.02 \pm 0.01_{A}^{a}$ & $0.01 \pm 0.01_{\mathrm{A}}^{\mathrm{a}}$ & $0.01 \pm 0.01_{\mathrm{A}}^{\mathrm{a}}$ \\
\hline $\mathrm{Na}$ & $1219.81 \pm 11.88_{\mathrm{A}}^{\mathrm{a}}$ & $1276.04 \pm 0.95_{\mathrm{A}}^{\mathrm{b}}$ & $915.2 \pm 118.09_{\mathrm{B}}^{\mathrm{a}}$ & $1342.66 \pm 5.13_{A}^{b}$ & $854.93 \pm 1.56_{B}{ }^{a}$ & $1338.48 \pm 47.43_{A}^{b}$ & $788.99 \pm 5.04_{B}{ }^{a}$ & $949.86 \pm 32.86_{\mathrm{B}}^{\mathrm{b}}$ \\
\hline $\mathrm{Ni}$ & $0.01 \pm 0.00_{\mathrm{A}}$ & ND & $0.11 \pm 0.01_{A}^{a}$ & $0.01 \pm 0.00_{\mathrm{A}}^{\mathrm{b}}$ & $0.08 \pm 0.04_{\mathrm{A}}^{\mathrm{a}}$ & $0.07 \pm 0.03_{\mathrm{A}}^{\mathrm{a}}$ & $0.10 \pm 0.06_{\mathrm{A}}^{\mathrm{a}}$ & $0.07 \pm 0.04_{A}^{a}$ \\
\hline $\mathrm{P}$ & $940.15 \pm 44.21_{\mathrm{A}}^{\mathrm{a}}$ & $849.69 \pm 51.71_{\mathrm{A}}^{\mathrm{a}}$ & $1156.1 \pm 37.96_{\mathrm{B}}^{\mathrm{a}}$ & $1524.56 \pm 67.36_{B}^{\circ}$ & $1038.74 \pm 60.43_{\mathrm{AB}}{ }^{\mathrm{a}}$ & $1283.02 \pm 10.75_{C^{b}}^{b}$ & $941.16 \pm 33.77_{\mathrm{A}}{ }^{\mathrm{a}}$ & $967.29 \pm 8.22_{\mathrm{A}}{ }^{\mathrm{a}}$ \\
\hline $\mathrm{Pb}$ & $0.25 \pm 0.03_{\mathrm{A}}^{\mathrm{a}}$ & $0.43 \pm 0.10_{\mathrm{A}}^{\mathrm{a}}$ & $0.34 \pm 0.08_{\mathrm{A}}^{\mathrm{a}}$ & $0.70 \pm 0.36_{A}^{a}$ & $0.35 \pm 0.11_{\mathrm{A}}^{\mathrm{a}}$ & $0.55 \pm 0.14_{\mathrm{A}}^{\mathrm{a}}$ & $0.27 \pm 0.05_{\mathrm{A}}^{\mathrm{a}}$ & $0.32 \pm 0.05_{\mathrm{A}}^{\mathrm{a}}$ \\
\hline $\mathrm{Se}$ & $0.29 \pm 0.08_{\mathrm{A}}^{\mathrm{a}}$ & $0.47 \pm 0.06_{\mathrm{A}}^{\mathrm{a}}$ & $0.42 \pm 0.00_{\mathrm{AB}}^{\mathrm{a}}$ & $0.75 \pm 0.00_{\mathrm{B}}^{\mathrm{b}}$ & $0.56 \pm 0.06_{\mathrm{B}}^{\mathrm{a}}$ & $0.56 \pm 0.01_{\mathrm{A}}^{\mathrm{a}}$ & $0.43 \pm 0.01_{\mathrm{AB}}{ }^{\mathrm{a}}$ & $0.56 \pm 0.04_{\mathrm{A}}^{\mathrm{b}}$ \\
\hline $\mathrm{Zn}$ & $46.6 \pm 2.31_{\mathrm{A}}^{\mathrm{a}}$ & $43.86 \pm 6.59{ }_{A}^{a}$ & $46.85 \pm 0.80_{\mathrm{A}}{ }^{\mathrm{a}}$ & $72.59 \pm 2.53_{B}^{b}$ & $50.57 \pm 0.32_{\mathrm{A}}{ }^{\mathrm{a}}$ & $66.47 \pm 1.66_{\mathrm{BC}}^{\mathrm{b}}$ & $49.42 \pm 1.00_{\mathrm{A}}{ }^{\mathrm{a}}$ & $54.56 \pm 3.29{ }_{\mathrm{C}}^{\mathrm{a}}$ \\
\hline
\end{tabular}

Different subscript upper case letters $(A, B, C)$ in the same row represent significant differences $(p<0.05)$ among different seasons for the same type of minerals in males or females. Different superscript lower case letters $(a, b)$ in the same row represent significant differences $(p<0.05)$ between males and females for same type of minerals in the same season. 
Table 2. Mineral content of marine mollusc/crustacean species from different studies $\left(\mathrm{mg} \mathrm{kg}^{-1}\right)$

\begin{tabular}{|c|c|c|c|c|c|c|c|c|c|c|}
\hline Authors & $\begin{array}{l}\text { Topcuoglu } \\
\text { et al. }(2002)\end{array}$ & $\begin{array}{l}\text { Gokoglu and } \\
\text { Yerlikaya, } 2003\end{array}$ & $\begin{array}{l}\text { Demopoulos } \\
\text { et al. (2008) }\end{array}$ & $\begin{array}{l}\text { Bat et al. } \\
(2009)\end{array}$ & $\begin{array}{l}\text { Barrento } \\
\text { et al. (2009) }\end{array}$ & $\begin{array}{l}\text { Bilgin and } \\
\text { Fidanbas } \\
(2011)\end{array}$ & $\begin{array}{l}\text { Mutlu } \\
\text { et al. (2011) }\end{array}$ & $\begin{array}{l}\text { Bordon } \\
\text { et al. }(2012)\end{array}$ & $\begin{array}{l}\text { Julshamn } \\
\text { et al. }(2015)\end{array}$ & $\begin{array}{l}\text { Present } \\
\text { study }\end{array}$ \\
\hline Location & Rize & Antalya & $\begin{array}{l}\text { Island of } \\
\text { Kosrae }\end{array}$ & Sinop & $\begin{array}{l}\text { Scottish } \\
\text { coast }\end{array}$ & Egirdir & $\begin{array}{l}\text { Mediterranean } \\
\text { Lagoon }\end{array}$ & $\begin{array}{l}\text { Santos } \\
\text { Estuarine } \\
\text { System }\end{array}$ & $\begin{array}{l}\text { Barents } \\
\text { Sea }\end{array}$ & Rize \\
\hline Species & $\begin{array}{l}\text { Rapana } \\
\text { venosa }\end{array}$ & $\begin{array}{l}\text { Callinectes } \\
\text { sapidus }^{*} \\
\text { Portunus } \\
\text { pelagicus }\end{array}$ & $\begin{array}{l}\text { Scylla } \\
\text { serrata }\end{array}$ & $\begin{array}{l}\text { Eriphia } \\
\text { verrucosa }\end{array}$ & $\begin{array}{l}\text { Cancer } \\
\text { pagurus }\end{array}$ & $\begin{array}{l}\text { Potamon } \\
\text { potamios }\end{array}$ & $\begin{array}{l}\text { Callinectes } \\
\text { sapidus }\end{array}$ & $\begin{array}{l}\text { Callinectes } \\
\text { danae }\end{array}$ & $\begin{array}{l}\text { Paralithodes } \\
\text { camtschaticus }\end{array}$ & $\begin{array}{l}\text { Eriphia } \\
\text { verrucosa }\end{array}$ \\
\hline $\mathrm{Al}$ & & & & & & & $1.2-13.7$ & $25.1-58.7$ & & $1.86-3.35$ \\
\hline B & & & $6.70-19.89$ & & & & & & & $2.17-3.65$ \\
\hline $\mathrm{Ca}$ & & $\begin{array}{l}649-1492^{*} \\
876-1509\end{array}$ & $3005.8-6893.4$ & & $\begin{array}{l}240-9430(\mathrm{M}) \\
200-2850(\mathrm{~F})\end{array}$ & $\begin{array}{l}8970-13530(\mathrm{M}) \\
9920-17870(\mathrm{~F})\end{array}$ & & & & 201.85-1419.19 \\
\hline $\mathrm{Cd}$ & $<0.02$ & & & 0.18 & $\begin{array}{l}\text { ND-28 (M) } \\
\text { ND-20 (F) }\end{array}$ & & $0.03-0.08$ & $0.012-0.022$ & $0.001-0.26$ & $0.02-0.05$ \\
\hline $\mathrm{Cr}$ & $<0.06$ & & & & & & $0.05-0.13$ & $0.005-0.500$ & & $0.05-0.35$ \\
\hline $\mathrm{Cu}$ & 15.61 & $\begin{array}{l}25.3-31.3^{*} \\
14.9-20.8\end{array}$ & $17.79-48.29$ & 2.61 & $\begin{array}{l}6.7-18(\mathrm{M}) \\
8.6-47(\mathrm{~F})\end{array}$ & $\begin{array}{l}50-70(\mathrm{M}) \\
50-60(\mathrm{~F})\end{array}$ & $5.38-11.7$ & $3.5-20.1$ & & $4.73-8.03$ \\
\hline $\mathrm{Fe}$ & 26.73 & $\begin{array}{l}10.4-11.3^{*} \\
4.5-6.8\end{array}$ & $47.47-78.74$ & 2.54 & $\begin{array}{l}3.2-23(\mathrm{M}) \\
3.7-18(\mathrm{~F})\end{array}$ & $\begin{array}{l}20-50(\mathrm{M}) \\
30-60(\mathrm{~F})\end{array}$ & $21.1-38.2$ & $6-21$ & & $2.80-6.44$ \\
\hline K & & $\begin{array}{l}2444-2563^{*} \\
3038-3089\end{array}$ & $11338-16812$ & & $\begin{array}{l}1670-3030(\mathrm{M}) \\
1620-3050(\mathrm{~F})\end{array}$ & $\begin{array}{l}10760-11700(\mathrm{M}) \\
8930-10440(\mathrm{~F})\end{array}$ & & & & $\begin{array}{l}1060.67- \\
1961.83\end{array}$ \\
\hline $\mathrm{Mg}$ & & $\begin{array}{l}351-371^{*} \\
488-558\end{array}$ & $2065.9-2673.5$ & & $\begin{array}{l}210-500(\mathrm{M}) \\
300-390(\mathrm{~F})\end{array}$ & $\begin{array}{l}1660-2080(\mathrm{M}) \\
1710-1860(\mathrm{~F})\end{array}$ & & & & $156.84-291.94$ \\
\hline $\mathrm{Mn}$ & 0.97 & $\begin{array}{l}3.7-3.9^{*} \\
0.6-1.6\end{array}$ & $2.55-4.07$ & 0.17 & $\begin{array}{l}0.26-2.1(\mathrm{M}) \\
0.24-2(\mathrm{~F})\end{array}$ & & $0.15-2.98$ & $0.8-5.6$ & & $0.32-1.93$ \\
\hline Mo & & & & & & & & & & $0.01-0.02$ \\
\hline $\mathrm{Na}$ & & $\begin{array}{l}2668-3269^{*} \\
3198-3535\end{array}$ & $13885-17357$ & & $\begin{array}{l}2240-3540(\mathrm{M}) \\
2230-3510(\mathrm{~F})\end{array}$ & $\begin{array}{l}9500-11590(\mathrm{M}) \\
9760-77880(\mathrm{~F})\end{array}$ & & & & $788.99-1342.66$ \\
\hline $\mathrm{Ni}$ & 1.57 & & & 1.42 & & & $0.24-0.45$ & $0.00-0.03$ & & $0.01-0.11$ \\
\hline $\mathrm{P}$ & $<0.5$ & $\begin{array}{l}1352-1654^{*} \\
1206-1542\end{array}$ & $8889.5-9973.5$ & & & $\begin{array}{l}740-900(\mathrm{M}) \\
760-950(\mathrm{~F})\end{array}$ & & & & $849.69-1524.56$ \\
\hline $\mathrm{Pb}$ & & & & 0.44 & $\begin{array}{l}\text { ND-0.03 (M) } \\
\text { ND-0.03 (F) }\end{array}$ & & & $0.003-1.725$ & $<0.01$ & $0.25-0.70$ \\
\hline $\mathrm{Se}$ & & & & & $\begin{array}{l}1-1.5(\mathrm{M}) \\
0.83-1.9(\mathrm{~F})\end{array}$ & & & & & $0.29-0.75$ \\
\hline $\mathrm{Zn}$ & 18.44 & $\begin{array}{l}47-69.9^{*} \\
37.2-46.8\end{array}$ & $286.49-335.55$ & 10.1 & $\begin{array}{l}17-74(\mathrm{M}) \\
22-70(\mathrm{~F})\end{array}$ & $\begin{array}{l}200-210(\mathrm{M}) \\
180-230(\mathrm{~F})\end{array}$ & $13.9-20.1$ & $20.1-33.8$ & & $43.86-72.59$ \\
\hline
\end{tabular}

M: Male, F: Female, ND: Not detected.

Table 3. Limit values of certain minerals in crustacean meat as per National and International Standards (wet weight basis)

\begin{tabular}{|c|c|c|c|c|c|c|c|c|c|c|c|c|c|c|c|c|c|}
\hline \multirow{2}{*}{ References } & \multicolumn{17}{|c|}{ Minerals } \\
\hline & $\mathrm{Al}$ & $\mathrm{B}$ & $\mathrm{Ca}$ & $\mathrm{Cd}$ & $\mathrm{Cr}$ & $\mathrm{Cu}$ & $\mathrm{Fe}$ & $\mathrm{K}$ & $\mathrm{Mg}$ & $\mathrm{Mn}$ & Mo & $\mathrm{Na}$ & $\mathrm{Ni}$ & $\mathrm{P}$ & $\mathrm{Pb}$ & $\mathrm{Se}$ & $\mathrm{Zn}$ \\
\hline $\begin{array}{l}\text { TKB. KKGM. Seafood quality control } \\
\text { handbook }(2000)\left(\mathrm{mg} \mathrm{kg}^{-1}\right)\end{array}$ & - & - & - & 1 & - & 20 & - & - & - & - & - & - & - & - & 2 & - & 50 \\
\hline Turkish food codex legislation (2002) $\left(\mathrm{mg} \mathrm{kg}^{-1}\right)$ & - & - & - & 0.5 & - & 20 & - & - & - & - & - & - & - & - & 0.5 & - & 50 \\
\hline Turkish food codex legislation (2011) $\left(\mathrm{mg} \mathrm{kg}^{-1}\right)$ & - & - & - & 0.5 & - & - & - & - & - & - & - & - & - & - & 0.5 & - & - \\
\hline FDA (2007) (ppm) & - & - & - & 3 & 12 & - & - & - & - & - & - & - & 70 & - & 1.5 & - & - \\
\hline $\mathrm{EC}(2007)\left(\mathrm{mg} \mathrm{kg}^{-1}\right)$ & - & - & - & 0.5 & - & - & - & - & - & - & - & - & - & - & 0.5 & - & - \\
\hline FSANZ (2005) $\left(\mathrm{mg} \mathrm{kg}^{-1}\right)$ & - & - & - & - & - & - & - & - & - & - & - & - & - & - & - & - & $25-40$ \\
\hline $\begin{array}{l}\text { FAO (Nauen, 1983) } \\
\text { (legal limits in countries) (ppm) }\end{array}$ & - & - & - & $0.05-5.5$ & 1 & $10-100$ & - & - & - & - & - & - & - & - & $0.5-10$ & $0.30-2$ & $30-1000$ \\
\hline
\end{tabular}


Table 4. The consumption limit values of certain minerals as per International standards (wet weight basis)

\begin{tabular}{|c|c|c|c|c|c|c|c|c|c|c|c|c|c|c|c|c|c|}
\hline \multirow{2}{*}{ References } & \multicolumn{17}{|c|}{ Minerals } \\
\hline & $\mathrm{Al}$ & B & $\mathrm{Ca}$ & $\mathrm{Cd}$ & $\mathrm{Cr}$ & $\mathrm{Cu}$ & $\mathrm{Fe}$ & K & $\mathrm{Mg}$ & $\mathrm{Mn}$ & Mo & $\mathrm{Na}$ & $\mathrm{Ni}$ & $\mathrm{P}$ & $\mathrm{Pb}$ & $\mathrm{Se}$ & $\mathrm{Zn}$ \\
\hline ATSDR (MRLs) & 1.0 & 0.2 & & 0.0003 & 0.005 & 0.01 & & & & 0.0003 & & & 0.0002 & & & 0.005 & 0.3 \\
\hline $\begin{array}{l}\text { (2013): } \\
\text { Oral }\left(\mathrm{mg} \mathrm{kg} \mathrm{day}^{-1}\right) \\
\text { Inhalation }\left(\mathrm{mg} \mathrm{m}^{-3}\right)\end{array}$ & Or. & Or. & & Or. Av. & Or. & Or. & & & & Inh. & & & Inh. & & & Or. & Or. \\
\hline $\begin{array}{l}\operatorname{EPA}(2000) \\
(\mathrm{mg} \mathrm{kg} \mathrm{day-1})\end{array}$ & & & & 0.001 & & & & & & & & & & & & 0.005 & \\
\hline $\begin{array}{l}\text { China Nutrition } \\
\left.\text { Institute (2001) (mg day }{ }^{-1}\right) \\
\text { Chen et al.(2007) }\end{array}$ & & & 800 & & & 1.0 & 12 & 1500 & 150 & 3.5 & & 900 & & 500 & & & 12 \\
\hline RDA $\left(m g\right.$ day $\left.^{-1}\right)(\mathrm{NAS}, 1989)$ & & & & & & 0.9 & $\begin{array}{l}8 \\
(\mathrm{M} \& \mathrm{~F}>51 \mathrm{yrs}) \\
18 \text { (F 19-50 yrs) }\end{array}$ & & & & 0.045 & & & & & 0.055 & $\begin{array}{l}11(\mathrm{M}) \\
8(\mathrm{~F})\end{array}$ \\
\hline $\mathrm{AI}$ (ages $19-50 \mathrm{mg}$ day ${ }^{-1}$ ) & & & & & $\begin{array}{l}0.035(\mathrm{M}) \\
0.025(\mathrm{~F})\end{array}$ & & & & & $\begin{array}{l}2.3(\mathrm{M}) \\
1.8(\mathrm{~F})\end{array}$ & & & & & & & \\
\hline $\begin{array}{l}\text { The U.S. Food and } \\
\text { Nutrition Board of the } \\
\text { Institute of Medicine } \\
\text { (Goldhaber, 2003) }\end{array}$ & & & & & & & & & & & & & & & & & \\
\hline $\begin{array}{l}\text { JECFA }\left(\mathrm{mg} \mathrm{kg} \mathrm{day}^{-1}\right) \\
\text { FAO/WHO }(1978 ; \\
\text { 1982; 1983; 1989) }\end{array}$ & 1.0 & & & 0.001 & & 0.5 & 0.8 & & & & & & & & 0.0035 & & 1.0 \\
\hline $\begin{array}{l}\operatorname{FDA}(2013) \\
\left(\mathrm{mg} \mathrm{day}^{-1}\right)\end{array}$ & & & 1000 & & 0.12 & 2 & 18 & 3.500 & 400 & 2 & 0.075 & 2400 & & 1000 & & 0.07 & 15 \\
\hline
\end{tabular}

Av: Average, M: Male, F: Female. Or: Oral, Inh: Inhalation

\section{Calcium (Ca)}

Daily intake of $\mathrm{Ca}$, which has an important place in human diet, has been specified by China National Institute (Chen et al., 2007) and FDA (2013) as $800 \mathrm{mg}$ and $1000 \mathrm{mg}$, respectively. The highest $\mathrm{Ca}$ content of $1419.19 \mathrm{mg} \mathrm{kg}^{-1}$ was recorded in male crabs during the present study in summer. The lowest value $\left(201.85 \mathrm{mg} \mathrm{kg}^{-1}\right)$ was found again in males in winter. Our results were found to be within the range reported in Cancer pagurus (200-17870 $\mathrm{mg} \mathrm{kg}^{-1}$ ) by Barrento et al. (2009) and lower than that reported by Bilgin and Fidanbas (2011) in Potamon potamios. Although some of our values seem to be high in terms of daily consumption, the differences are not expected to pose a risk to human health.

\section{Cadmium (Cd)}

Highest $\mathrm{Cd}$ content was recorded for females (0.05 mg kg-1) in winter, while the lowest value of $0.02 \mathrm{mg} \mathrm{kg}^{-1}$ was recorded in males during spring. $\mathrm{Cd}$ values observed by us were within the consumption values given by EPA (2000) and JECFA (1989), while the levels were found to exceed the limits specified by ATSDR (2013), considering average human weight of $60-70 \mathrm{~kg}$. Cd contents recorded in our study were found to fall within the range as reported in literature (Barrento et al., 2009; Julshamn et al., 2015).

\section{Chromium $(\mathrm{Cr})$}

In the present study; the highest value of $\mathrm{Cr}$ was determined in male crabs at $0.35 \mathrm{mg} \mathrm{kg}^{-1}$ in winter and the lowest value of $0.05 \mathrm{mg} \mathrm{kg}^{-1}$ was recorded during spring in both sexes. The values were found to be within the range (0.005- $0.5 \mathrm{mg} \mathrm{kg}^{-1}$ ) reported by Bordon et al. (2012). The $\mathrm{Cr}$ content of warty crab recorded during all the seasons were found to be below the limit value specified by FDA (2007) and FAO (Nauen,1983). However, the values of male and female crabs were found to be above the daily consumption levels specified by the U.S. Food and Nutrition Board of the Institute of Medicine (Goldhaber, 2003).

\section{Copper (Cu)}

The highest $\mathrm{Cu}$ value was observed in females (8.03 $\left.\mathrm{mg} \mathrm{kg}{ }^{-1}\right)$ in autumn and the lowest value $\left(4.75 \mathrm{mg} \mathrm{kg}^{-1}\right)$ again in females in winter and the values were within the ranges reported by Bat et al. (2009) and Bilgin and Fidanbaş (2011). It was found that the limit values specified in Table 3 did not exceed our values. With respect to the daily consumption, results of the present study were found to be above all the specified limit values except those by JECFA (1989).

Iron $(\mathrm{Fe})$

Iron was found to be lowest in males $(2.64 \mathrm{mg}$ $\left.\mathrm{kg}^{-1}\right)$ in summer and the highest value of $6.44 \mathrm{mg} \mathrm{kg}^{-1}$ 
was found in females in spring. In previous reports, $\mathrm{Fe}$ content ranged between 2.54-78.74 $\mathrm{mg} \mathrm{kg}^{-1}$ (Demopoulos et al., 2008; Bat et al., 2009). The values obtained in the present study are within this range. The Fe content determined in the present study was found to be under the recommended dietary allowance (RDA) set by NAS (1989).

\section{Potassium (K)}

The highest amount of $\mathrm{K}\left(1961.83 \mathrm{mg} \mathrm{kg}^{-1}\right)$ was found in females during summer and the lowest (1060.67 $\mathrm{mg} \mathrm{kg}^{-1}$ ) in males again in summer. Potassium content in the range between $1620-16812 \mathrm{mg} \mathrm{kg}^{-1}$ has been rported in various studies (Demopoulos et al., 2008; Barrento et al., 2009). With respect to daily consumption, the potassium levels in warty crab recorded during different seasons for males and females in the present study, except those for summer-female and autumn-female were found to be below the values specified by China Nutrition Institute (Chen et al., 2007) and FDA (2013).

\section{Magnesium (Mg)}

Highest value of $291.94 \mathrm{mg} \mathrm{kg}^{-1}$ was measured in females in summer and the lowest $\left(156.84 \mathrm{mg} \mathrm{kg}^{-}\right.$ ${ }^{1}$ ) in males in winter. These values were found to be less than the $\mathrm{Mg}$ contents content (300-390 $\mathrm{mg} \mathrm{kg}{ }^{-1}$ ) of female Canger pagurus reported by Barrento et al. (2009), while these were within range of 210-500 $\mathrm{mg} \mathrm{kg}^{-1}$ found in males. In terms of daily consumption values, data of the present study were found to be above the values specified by China Nutrition Institute (Chen et al., 2007), but below those specified by FDA (2013).

\section{Manganese (Mn)}

The highest Mn content (1.93 $\left.\mathrm{mg} \mathrm{kg}^{-1}\right)$ was observed in females in spring while the lowest value (0.32 $\mathrm{mg} \mathrm{kg}^{-1}$ ) was found in males in winter. The amount of $\mathrm{Mn}$ was determined to be between 0.15-5.6 $\mathrm{mg} \mathrm{kg}^{-1}$ (Mutlu et al., 2011; Bordon et al., 2012). With respect to the daily consumption limits specified for $\mathrm{Mn}$, the content of $\mathrm{Mn}$ in warty crab was found to be within the limit values specified by the U.S. Food and Nutrition Board of the Institute of Medicine (Goldhaber, 2003). The limit specified by ATSDR was not assessed as it is expressed in terms of inhalation.

\section{Molybdenum (Mo)}

In the analyses performed with respect to season and gender; Mo content in the range of 0.01-0.02 $\mathrm{mg} \mathrm{kg}^{-1}$ were recorded. No previous studies specified the limit for Mo content in seafood. Examining the daily consumption values, the data from the present study were found to be below the values specified by FDA (2013).

\section{Sodium $(\mathrm{Na})$}

While $\mathrm{Na}$ content in females in summer (1342.66 $\mathrm{mg} \mathrm{kg}^{-1}$ ) indicated the highest level, the lowest (788.99 $\mathrm{mg} \mathrm{kg}^{-1}$ ) was observed in males in winter. The results from the present study were observed to be lower than the the range of $2230-77880 \mathrm{mg} \mathrm{kg}^{-1}$ reported by Barrento et al. (2009) and Bilgin and Fidanbas (2011). Our results, were found to be above the daily consumption values specified by China Nutrition Institute (Chen et al., 2007) while, below the limits specified by FDA (2013).

\section{Nickel (Ni)}

While the amount of $\mathrm{Ni}$ was found to be below the detection limits in females, the highest levels were measured in males $\left(0.11 \mathrm{mg} \mathrm{kg}^{-1}\right)$ in summer. Ni amounts were determined to be between $0.00-1.57 \mathrm{mg} \mathrm{kg}^{-1}$ in the previous studies (Topcuoglu et al., 2002; Bordon et al., 2012). Also, the results of the present study were found to comply with both limit value standards and daily consumable amount standards.

\section{Phosphorus $(P)$}

The highest value was measured in females $\left(1524.56 \mathrm{mg} \mathrm{kg}^{-1}\right)$ in summer and the lowest value again in females $\left(849.69 \mathrm{mg} \mathrm{kg}^{-1}\right)$ during spring. The results of the present study were observed to fall within the range of $740-9973.5 \mathrm{mg} \mathrm{kg}^{-1}$ reported by Bilgin and Fidanbas, (2011) and Demopoulos et al., (2008). All of our data were found to be higher than the values specified by China Nutrition Institute (Chen et al., 2007) in terms of daily consumption amounts and some of them were found to be higher than the value specified by FDA (2013).

\section{Lead $(\mathrm{Pb})$}

The highest value for $\mathrm{Pb}$ was measured in females at $0.70 \mathrm{mg} \mathrm{kg}^{-1}$ in summer and the lowest value in males $\left(0.25 \mathrm{mg} \mathrm{kg}^{-1}\right)$ in spring. All the values in the present study were found within the range of 0.003 to 1.725 $\mathrm{mg} \mathrm{kg} \mathrm{k}^{-1}$ of lead as reported by Bordon et al. (2012). $\mathrm{Pb}$ amounts of both male and female were found below national and international standard values (Table 3), except for those of the female crabs in summer and autumn. The data in the present study were found to be higher than the daily consumable amount of 0.0035 mg kg-1 ${ }^{-1}{ }^{-1}$ specified by JECFA:

\section{Selenium $(\mathrm{Se})$}

The highest amount of selenium was found in females at $0.75 \mathrm{mg} \mathrm{kg}^{-1}$ in summer and the lowest amount in males $\left(0.29 \mathrm{mg} \mathrm{kg}^{-1}\right)$ in spring. The Se values obtained were lower than the range of $0.83-1.9 \mathrm{mg} \mathrm{kg}^{-1}$ reported by Barrento et al. (2009). In the regulation specified by FAO 
(Nauen, 1983), where limit values of several countries were given, the maximum value for Se was specified as $2 \mathrm{mg} \mathrm{kg}^{-1}$. Examining Table 4, in which daily consumption amounts are given, it is seen that the data of the present study were above the specified values except for ATSDR (2013) and EPA (2000).

\section{Zinc (Zn)}

In the present study; the highest value of $\mathrm{Zn}$ was found in females $\left(72.59 \mathrm{mg} \mathrm{kg}^{-1}\right)$ in summer and the lowest in males (43.86 $\mathrm{mg} \mathrm{kg}^{-1}$ ) in spring. $\mathrm{Zn}$ values from earlier studies varied in the range of 10.1-335.55 mg kg-1 (Demopoulos et al., 2008; Bat et al., 2009). Our data were found to be higher than the limit values (25-40 $\mathrm{mg} \mathrm{kg}^{-1}$ ) specified by FSANZ (2005) and the values in summer, autumn and winter in females and in autumn in male were found to be higher than the limit value (50 mg kg ${ }^{-1}$ ) specified by TKB (2000) and Turkish Food Codex Legislation (2002). In terms of seasonal and gender differences, our results were found to be above the daily consumable limit value. Since the benefits of zinc and inadequate consumption by the Turkish people (Taneli, 2005) are well-known, it is expected that the consumption and limit values might not pose a problem considering the crab consumption habits.

In the present investigation, the levels of $\mathrm{B}, \mathrm{Na}$ and $\mathrm{Se}$ recorded in warty crab were found to be lower than those reported in earlier studies. $\mathrm{Al}, \mathrm{Ca}, \mathrm{Cd}, \mathrm{Cr}, \mathrm{Cu}$, $\mathrm{Fe}, \mathrm{K}, \mathrm{Mg}, \mathrm{Mn}, \mathrm{Ni}, \mathrm{P}, \mathrm{Pb}$ and $\mathrm{Zn}$ levels were found to be with in the range reported in other studies.. In terms of the limit values specified in national and international standards; it was found that $\mathrm{Al}, \mathrm{B}, \mathrm{Ca}, \mathrm{Cd}, \mathrm{Cr}, \mathrm{Cu}, \mathrm{Fe}$, $\mathrm{K}, \mathrm{Mg}, \mathrm{Mn}, \mathrm{Mo}, \mathrm{Na}, \mathrm{Ni}, \mathrm{P}$ and Se levels recorded in the present study did not exceed the limit values; but $\mathrm{Pb}$ and $\mathrm{Zn}$ values were found to be above the limits. In terms of daily consumption values; $\mathrm{Al}, \mathrm{B}, \mathrm{Fe}, \mathrm{K}, \mathrm{Mn}, \mathrm{Mo}$ and $\mathrm{Ni}$ levels were found to be within the limit as specified by all standards. It was also observed that $\mathrm{Ca}, \mathrm{Cd}, \mathrm{Cu}$, $\mathrm{Mg}$, $\mathrm{Na}$ and $\mathrm{P}$ levels were above certain standard values; while $\mathrm{Cr}, \mathrm{Pb}$, Se and $\mathrm{Zn}$ levels were above the limit values specified by all standards. Fourteen minerals were found higher in females while 3 minerals were higher in males. Seasonally, mineral content were observed to be higher in summer, and lower during winter and autumn. A balanced diet of crab meat in Rize Province is expected to eliminate the mineral deficiencies in humans.

\section{References}

ATSDR 2013. U. S. Department of health and human services, Public Health Services. Agency for Toxic Substances and Disease Registry, Atlanta, GA, USA.
Barrento, S., Marques, A., Teixeira, B., Carvalho, M. L., Vaz-Pires, P. and Nunes, M. L. 2009. Influence of season and sex on the contents of minerals and trace elements in brown crab (Cancer pagurus Linnaeus, 1758). J. Agri. Food Chem. 57(8): 3253-60.

Bat, L., Gokkurt, O., Sezgin, M., Ustun, F. and Sahin, F. 2009. Evaluation of the Black Sea land based sources of pollution in the coastal region of Turkey. The Open Mar. Biol. J. 3: $112-24$.

Bernhard, M. 1976. Manual of methods in aquatic environment research, FAO Fisheries Technical Paper, No 158 FIRI/T 158, Food and Agricultural Organisation, Rome, 123 pp.

Bilgin, S. and Fidanbas, Z. U. C. 2011. Nutritional properties of crab (Potamon potamios Olivier, 1804) in the Lake of Egirdir (Turkey). Pakistan Vet. J., 31(3): 239-43.

Bordon, I. C. A. C., Sarkis, J. E. S., Tomas, A. R. G., Scalco, A., Lima, M., Hortellani, M. A. and Andrade, N. P. 2012. Assessment of metal concentrations in muscles of the blue crab, Callinectes danae S., from the Santos Estuarine System. Bull. Environ. Cont. Toxicol., 89(3): 484-8.

Chen, D. W., Zhang, M. and Shrestha, S. 2007. Compositional characteristics and nutritional quality of Chinese mitten crab (Eriocheir sinensis). Food Chem., 103: 1343-9.

Caglak, E. and Karsl1, B. 2014. Investigation of some heavy metals accumulation in muscle of pike perch (Stizostedion lucioperca, Linnaeus 1758) from Lake Beysehir, Turkey. $J$. Agric. Sci., 20: 203-14.

Demopoulos, A. W. J., Cormier, N., Ewel, K. C. and Fry, B. 2008. Use of multiple chemical tracers to define habitat use of Indo-Pacific mangrove crab, Scylla serrata (Decapoda: Portunidae). Estuaries and Coasts, 31(2): 371-81.

EC 2007. Cadmium oxide and cadmium metal. European Union Risk Assessment Report, European Commission, Italy, $678 \mathrm{pp}$.

EPA 2000. Guidance for assessing chemical contaminant data for use in fish advisories. In: Cunningham, P. and Goldhaber, S. (Eds.), Risk assessment and fish consumption limits, $3^{\text {rd }}$ edn. United States Environmental Protection Agency, Office of Water (4305), 823-B-00-008, p. 158-170.

FDA 2007. National shellfish sanitation program guide for the control of molluscan shellfish, United States Food and Drug Administration, USA.

FDA 2013. Guidance for Industry: A food labeling guide (14. Appendix F: Calculate the Percent Daily Value for the Appropriate Nutrients). United States Food and Drug Administration, USA.

FSANZ 2005. Primary production and processing standard for seafood. Australian Government, Federal Register of Legislation, Gazette No. FSC 20. Final Assessment Report, 345 pp. 
Goldhaber, S. B. 2003. Trace element risk assessment: essentiality $v s$ toxicity. Regulatory toxicology and pharmacology, 38: $232-242$.

Gokoglu, N. and Yerlikaya, P. 2003. Determination of proximate composition and mineral contents of blue crab (Callinectes sapidus) and swimming crab (Portunus pelagicus) caught off the Gulf of Antalya. Food Chem., 80: 495-8.

JECFA 1978. Evaluation of certain food additives. Twenty-first report of the Joint FAO/WHO Expert Committee on Food Additives. WHO Technical Report Series, No. 617, World Health Organisation.

JECFA 1982. Evaluation of certain food additives and contaminants. Twenty-sixth report of the Joint FAO/WHO Expert Committee on Food Additives. WHO Technical Report Series, No. 683, World Health Organisation.

JECFA 1983. Evaluation of certain food additives and contaminants. Twenty-seventh report of the Joint FAO/WHO Expert Committee on Food Additives. WHO Technical Report Series, No. 696, World Health Organisation.

JECFA 1989. Evaluation of certain food additives and contaminants, (Thirty-third report of the Joint FAO/WHO Expert Committee on Food Additives. WHO Technical Report Series, No. 776, World Health Organisation.

Jimmy, U. P. and Arazu, V. N. 2012. The proximate and mineral composition of two edible crabs Callinectes amnicola and Uca tangeri (Crustecea: Decapoda) of The Cross River, Nigeria. Pakistan J. Nutr., 11(1): 78-82.

Julshamn, K., Valdersnes, S., Duinker, A., Nedreaas, K., Sundet, J. H. and Maage, A. 2015. Heavy metals and POPs in red king crab from the Barents Sea. Food Chem., 167: 409-17.

Kayhan, F. E., Koc, N. D., Muslu, M. N, and Colak, S. 2010. Izmit Korfezi'nden avlanan derin su pembe karidesi'nin (Parapenaeus longirostris Lucas, 1946) biyokimyasal kompozisyonu ve mineral iceriklerinin belirlenmesi. Kafkas Universitesi Veterinerlik Fakultesi Dergisi, 16: $189-96$

Kucukgelmez, A., Celik, M., Yanar, Y., Ersoy, B. and Cikrikci, M. 2006. Proximate composition and mineral contents of the blue crab (Callinectes sapidus) breast meat, claw meat and hepatopancreas. Int. J. Food Sci. Tech., 41: 1023-26.
Mutlu, C., Turkmen, M., Turkmen, A. and Tepe, Y. 2011. Comparison of metal concentrations in tissues of blue crab, Callinectes sapidus from Mediterranean lagoons. Bull. Environ. Cont. Toxicol., 87: 282-286.

NAS 1989. Recommended Dietary Allowances, $10^{\text {th }}$ edn. National Academy of Sciences, Food and Nutrition Board, Commission on Life Sciences, National Research Council, National Academy Press, Washington, DC, USA.

Nauen, C. E. 1983. Compilation of legal limits for hazardous substances in fish and fishery products. Food and Agriculture Organisation of the United Nations, FAO Fisheries Technical Paper. No. 764, FIRI/C764.

Sukender, K., Jaspreet, S., Sneha, D. and Munish, G. 2012. AAS estimation of heavy metals and trace elements in Indian herbal cosmetic preparations. Res. J. Chem. Sci., 2: 46-51.

Sumbuloglu, K. and Sumbuloglu, V. 2000. Biyoistatistik. Hatiboglu Press, Ankara, 269 pp.

Taneli, B. 2005. Anadolu Toplumunda Cinko. Ege Tip Dergisi, 44(1): 1-10.

TKB 2000. Tarım ve Koyisleri Bakanlig1, Koruma ve Kontrol Genel Mudurlugu, Seafood quality control handbook, Ankara, 198 pp.

Topcuoglu, S., Kırbaşoglu, C. and Gungor, N. 2002. Heavy metals in organisms and sediments from Turkish Coast of the Black Sea, 1997-1998. Environ. Int., 27: 521-526.

TUIK 2016. Fishery statistics. Turkish Statistical Institute, Ankara, Turkey.

Turkish Food Codex Legislation 2002. Gida maddelerinde belirli bulasanlarin maksimum seviyelerinin belirlenmesi hakkında teblig 23.09.2002-24885 Sayılı Resmi Gazete.

Turkish Food Codex Legislation 2011. Gida, Tarım ve Hayvancllik Bakanligl Turk Gida Kodeksi Bulasanlar Yonetmenligi, 29 Aralık 2011-28157 Sayıl1 Resmi Gazete.

Zhang, Z., He, L., Li, J. and Wu, Z. B. 2007. Analysis heavy metals of muscle and intestine tissue in fish-in Banan Section of Chongqing from Three Gorges Reservoir, China. Polish J. Environ. Stud., 16(6): 949-58. 